\title{
Common Variable Immunodeficiency at Adult Age
}

\author{
Muzaffer Senol ${ }^{1}$, Neslihan Ozcelik ${ }^{1}$, Songul Ozyurt ${ }^{1}$, ${ }^{D}$, Halil Donmez ${ }^{\mathbb{D}}$, Aziz Gumus ${ }^{1}$, Unal Sahin ${ }^{1}$ (D) \\ ${ }^{1}$ Recep Tayyip Erdogan University, Department of Chest Diseases, Rize, Turkey. \\ ${ }^{2}$ Recep Tayyip Erdogan University, Department of Allergy and Immunology, Rize, Turkey \\ Correspondence Author: Neslihan Ozcelik \\ E-mail: neslihan.ozcelik@erdogan.edu.tr \\ Received: 05.01.2021 Accepted: 24.04.2021
}

\begin{abstract}
Common Variable Immunodeficiency (CVID) is a heterogeneous group of diseases progressing with recurrent infections. This disease should be considered in cases experiencing recurrent lung infection, otitis media, rhinosinusitis, and urinary tract infection. Since it is generally seen in the childhood age group, the diagnosis in adults may be delayed. A Common Variable Immunodeficiency case, which was followed up in our clinic and characterized by recurrent upper respiratory tract infection and pneumonia, was presented to raise awareness on this issue. CVID should be kept in mind in patients who are admitted to outpatient clinics with recurrent infections, who do not respond despite appropriate treatments, and who develop complications.
\end{abstract}

Keywords: Adult, Common Variable Immunodeficiency, Infection, Pneumonia

\section{INTRODUCTION}

Common Variable Immunodeficiency (CVID) is a heterogeneous group of diseases progressing with recurrent infections [1]. It usually gives clinical symptoms within the first 20 years. It is prevalent in 10 years of age in childhood and $30-40$ years in adults. The gender distribution is equal. It is one of the most common immune deficiencies, and although genetic mutations have been identified, its formation mechanism is not fully known. Since patients are examined in different departments with different clinical presentations, diagnosis is often delayed. This disease should be considered in cases presenting with a recurrent lung infection, otitis media, rhinosinusitis, and urinary tract infection. The prevalence of the disease in our country is unknown. There is a lack of epidemiological data in this disease group due to the lack of awareness and knowledge of appropriate diagnostic methods.

In order to increase awareness on this issue, we present a late-diagnosed immunodeficiency case.

\section{CASE}

A 55-year-old male patient was admitted to the emergency department with cough, sputum, and fever complaints. Upon observation of consolidations, including air bronchogram and pleural effusion on computed lung tomography, the patient was hospitalized with a preliminary diagnosis of pneumonia (Figure 1). In his history, it was learned that he was under follow-up in another health facility for asthma, and he had been using inhaler treatment for five years and had no history of smoking. It was also learned that the patient had had otitis media several times in the last year with similar complaints and was hospitalized three times with a pneumonia diagnosis. When the previous radiological imagings were examined, displacement-like consolidations were observed. In order to identify the etiology, diagnostic thoracentesis and bronchoscopy procedures were performed. The procedure results ended up as consistent with benign cytology. The immunodeficiency pre-diagnosis was considered for the patient who had a history of frequent hospitalizations after eliminating the presence of malignancy. Serum immunoglobulins were low (IgG <108 $\mathrm{mg} / \mathrm{dL}, \operatorname{lgA}<5 \mathrm{mg} / \mathrm{dL}, \operatorname{lgM}<5 \mathrm{mg} / \mathrm{dL}$, anti-HIV: negative), other laboratory parameters were normal, the 23-serotype pneumococcal vaccine was administered, and there was no significant increase in pneumococcal antibody level after six weeks. The patient was diagnosed with common variable immunodeficiency (CVID), and intravenous immunoglobulin (IVIG) treatment was initiated. The patient is now under the follow-up of our Allergy and Immunology clinic. 


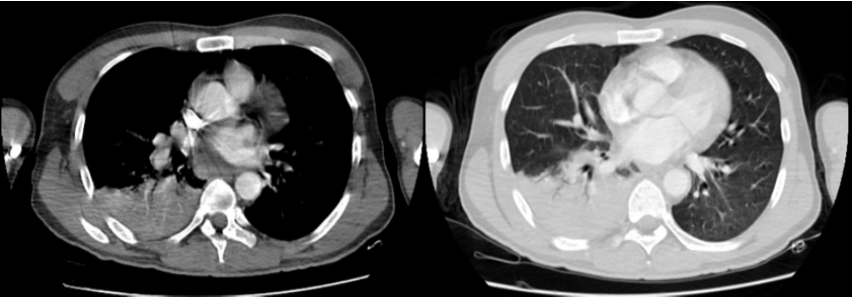

Figure 1. Computed tomography image shows consolidations including air bronchogram in the sections of mediastinum and parenchyma.

\section{DISCUSSION}

Common Variable Immune Deficiency (CVID) is characterized by a deficiency in immunoglobulin synthesis due to impaired $B$ cell proliferation. CVID is the roof for a large group of diseases that includes a variety of genetic disorders. It is the most common primary immunodeficiency condition in adults. The highest CVID prevalence was $40.2 \%$ of all primary immunodeficiency (PID) patients in the United States, while the lowest rates were in the Middle East countries at $2.6 \%$ and Africa at only 1.3\% [2]. However, it is known as the most common form of PID in the Caucasian population [3].

In various studies, the average age of onset of the disease is around 33 years in women and 28 in men $[1,4]$. Considering these studies, our patient was diagnosed at a more advanced age. The oldest patient reported in the literature is a 73-yearold female patient who was described in the study of Ardeniz et al.

The disease has been named 'variable' because of emerging with different presentations. Susceptibility to infection is the most common clinical finding in these patients. Infections such as sinusitis, pharyngitis, otitis, and especially pneumonia caused by encapsulated bacteria such as streptococcus pneumonia, staphylococcus aureus, Haemophilus influenza are common. Although rare, severe infections such as giardia diarrhea, sepsis, empyema, osteomyelitis, meningitis, septic arthritis can be seen. Our patient also had common sinopulmonary infections. Due to this clinical range and the fact that clinicians are not aware of this disease, the average diagnosis time reaches 6-7 years. Our patient applied to the hospital and outpatient clinic many times with asthma and pneumonia diagnosis in the last one year.

The disease may be accompanied by a sarcoidosis-like reaction with diffuse granulomas in the lung, spleen, and lymph nodes or autoimmune diseases with a rate of 25\% [5]. Autoimmune diseases such as hemolytic anemia, pernicious anemia, splenomegaly, primary biliary cirrhosis, uveitis, and psoriasis can also be seen frequently in the CVID clinic. Once again, the risk of malignancy, especially lymphomas, has increased in this disease, compared to the normal population [3]. Extranodal involvement is common, especially in nonHodgkin lymphoma.

The most common lung complication of CVID is the increased risk of pneumonia. Bronchiectasis and interstitial lung disease are two major lung complications that occur after recurrent and severe lung infections [2]. Our patient had recurring pneumonia with a displacing characteristic. There was bronchiectasis development (Figure 2); however, there was no interstitial involvement.

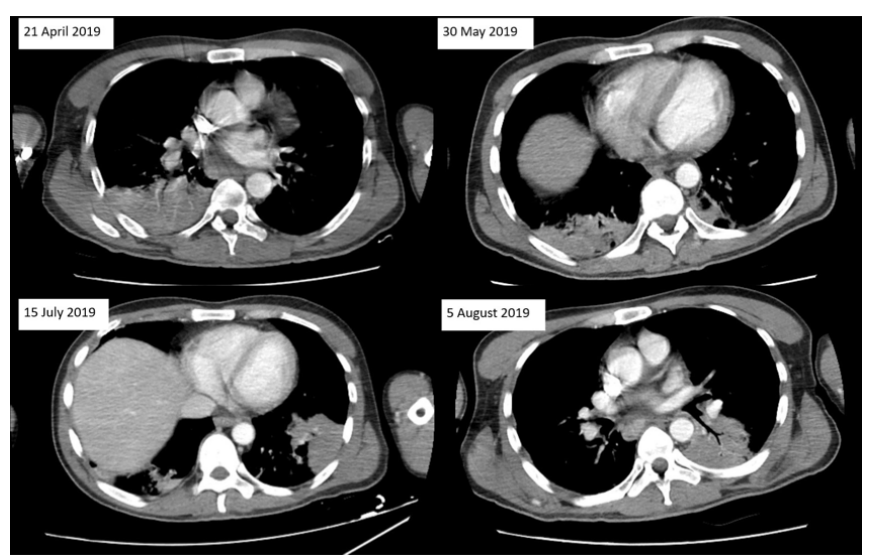

Figure 2. Computed tomography sections taken at different dates Show pneumonia in different localizations.

Diagnosis is made by demonstrating low serum immunoglobulin (Ig) A, G, M levels, and the absence or decrease of antibody production with laboratory tests. If the IgG level is below $150 \mathrm{mg} / \mathrm{dl}$, there is no need for additional tests. However, if the IgG is at the level of $400-600 \mathrm{mg} / \mathrm{dl}$ or if there is only a slight decrease in Ig A levels, additional tests are required. The lack of reaction to vaccines in patients should also be alarming for CVID, as it would suggest a decreased antibody response. Other diseases that may cause low immunoglobulin should be considered. In our case, the IgG level was below 108, and there was no antibody response to the pneumococcal vaccine.

In the treatment of CVID, $400-600 \mathrm{mg} / \mathrm{kg}$ intravenous immunoglobulin (IVIG) is recommended once a month. Apart from this, appropriate management and treatment of infections and complications should be accomplished. In our case, IVIG was initiated after appropriate antibiotic treatment for lung infection.

\section{CONCLUSION}

CVID should be kept in mind in patients who are admitted to outpatient clinics with recurrent infections, who do not respond despite appropriate treatments, and who develop complications.

\section{REFERENCES}

[1] Musabak UH, Demirel F, Yesillik S, Baysan A, Selcuk A, Kartal $\mathrm{O}$, et al. Adults with common variable immunodeficiency: a single center experience. Turkish Journal of Medical Sciences 2017; 47.1:1-12

[2] Yazdani R, Habibi S, Sharifi L, Azizi G, Abolhassani H, Olbrich $P$, et al. Common variable immunodeficiency: epidemiology, pathogenesis, clinical manifestations, diagnosis, classification, 
and management. Journal of Investigational Allergology and Clinical Immunology 2020;30:14-34.

[3] Cunningham-Rundles C. How I treat common variable immune deficiency. Blood 2010;116:7-15.

[4] Ardeniz O, Kacmaz Basoglu O, Gunsar F, Unsel M, Bayraktaroglu $\mathrm{S}$, Mete $\mathrm{N}$, et al. Clinical and immunological analysis of 23 adult patients with common variable immunodeficiency.
Journal of Investigational Allergology and Clinical Immunology 2010;20:222-236.

[5] Musaffa Salepci B, Diktas Tahtasakal S, Kiral N, Sener Comert $\mathrm{S}$, Sarac G, Caglayan B. Iki olgu nedeniyle ampiyem ile ortaya cikan yaygın degisken immun yetmezlik sendromu. Journal of Kartal Training and Research Hospital/Kartal Egitim ve Arastirma Hastanesi Tip Dergisi 2013;24:179-186. 